applied, specialized or interdisciplinary.

Nevertheless, Lock's survey and recommendations are welcome. They include a suggestion that in special cases it would be useful to complement closed peer review with the publication of open peer commentary. I cannot but second this, particularly as 39 of the 281 items in Lock's reference list come from a controversial peer-review study that appeared in the peer-commentary journal I edit - a study whose themes, Lock writes, "run through this monograph as a leit-motiv, almost as obsessionally (to mix my metaphors) as the Suez canal ran through Lady
Eden's drawing room during the 1956 Suez crisis".

There is an interesting parallel between beliefs about "the peer review system" and beliefs about "the scientific method". Those who favour proposition (i) tend to worry about biases and conspiracies that could impede the ideal march of progress. Those, like myself, who lean towards (ii), feel that it's more a matter of trying to reduce inefficiencies in yet another very human domain of endeavour.

Stevan Harnad is Editor of The Behaviora and Brain Sciences, 20 Nassau Street, Prince ton, New Jersey 08542, USA

\section{Hormonal activity}

\section{Michael J. Brownstein}

Neurobiology of Vasopressin. Edited by D. Ganten and D. Pfaff. Springer-Verlag: 1985. Pp.203. DM 98.

Vasopressin. Edited by Robert W. Schrier. Raven:1985. Pp.577. \$104.50.

THE history of research on vasopressin goes back almost a hundred years, to 1895, when Oliver and Schafer showed that mammalian pituitary extracts had pressor (blood-pressure-elevating activity). Three years later Howell demonstrated that the factor responsible resided in the posterior or neural lobe of the gland. Subsequently, Dale found that neural lobe extracts possessed oxytocic activity (that is, they contracted uterine smooth muscle) and von den Velden showed that these extracts could inhibit diuresis as well. In the course of the next 20 years it was established that the oxytocic and pressor-antidiuretic effects were mediated by separate factors, oxytocin and vasopressin, respectively. These were finally purified, characterized and chemically synthesized by du Vigneaud and his colleagues in the $1950 \mathrm{~s}$.

Early workers found it difficult to believe that the posterior pituitary could elaborate hormones. Cytologically it is very different from most glands. The revolutionary discovery by Bargmann and Scharrer in 1951 that the neural lobe is part of a neurosecretory system resolved this problem. The vasopressin in the neural lobe is actually in axons and nerve terminals provided by hypothalamic neurones; vasopressin is released from these nerve terminals into blood vessels to act on its peripheral targets.

Neurobiology of Vasopressin, the fourth book in a series on current topics in neuroendocrinology, contains five chapters contributed by a total of nine authors. It nicely outlines the research work on vasopressin of the past decade, a period during which many other neuropeptides have come under careful scrutiny. The amino-acid sequence of the vasopressin precursor is now known and its gene has been characterized. Cells that make it have been visualized immunocytochemically. (Many of these lie outside the nuclei that project to the posterior pituitary and seem to be involved in centra regulation of autonomic function; surprisingly, some vasopressin-positive cells are present in peripheral tissues such as the adrenal and ovaries.) Steps are being taken towards understanding how vasopressin's synthesis is regulated, and how the afferent inputs to the cells that manufacture it control its production and release in response to changes in water balance.
In spite of the fact that vasopressin's name derives from its effects on blood vessels, these effects are only seen at high dose levels and its cardiovascular effects attracted little interest for many years. This is no longer the case. It is now understood that vasopressin's vasoconstrictor effects are masked by some of its other actions: facilitation of the inhibitory actions of baroreceptive pathways on sympathetic vasomotor activity, and depression of renin secretion. Appropriately, therefore, a large part of Vasopressin, the second book reviewed here, is given over to descriptions of the hormone's interactions with central and peripheral baroreceptor systems and regional vascular beds. Also included are sections devoted to the cellular action of vasopressin, vasopressin antagonists, osmoregulation of drinking and vasopressin secretion, neural control of vasopressin release, and the role of vasopressin in producing pathological states.

While many of the individual chapters are very good, the overall organization of the book leaves something to be desired. Those new to the field would have profited from introductory descriptions of vasopressin's structure, biosynthesis, site of production and release, actions and receptors. It is only in the context of this background information that subsequent chapters, which deal with intricate physiological and pharmacological experiments, can be appreciated.

For this reason, I would suggest that readers should tackle Neurobiology of Vasopressin before attempting to digest its larger sister volume; in particular, the chapters on volume and cardiovascular regulation in Ganten and Pfaff's book will provide helpful overviews for those attempting to get their bearings on the vasopressin literature. Both books are worth studying, and that neither of them is quite up to date might be seen as a plus point; work on vasopressin is moving along very quickly.

Michael J. Brownstein is in the Laboratory of Cell Biology, National Institute of Mental Health, Bethesda, Maryland 20205, USA.

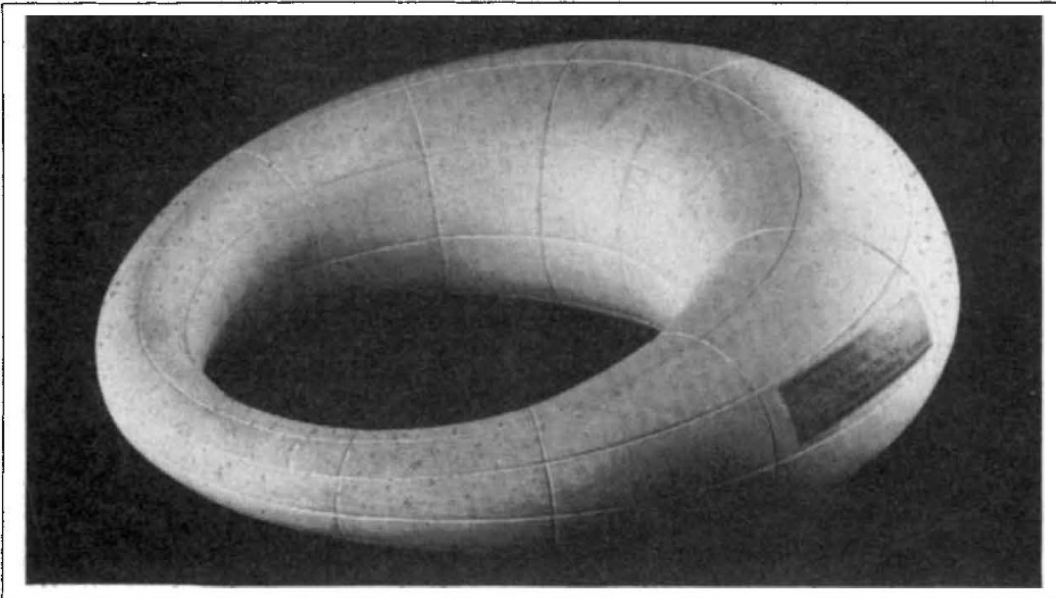

ONE of the pleasanter branches of nineteenth-century mathematics was the study of curves and surfaces in space. In order to examine and communicate their properties, geometers built three-dimensional models which frequently possess that interplay of simplicity and complexity, of order and chaos, which characterizes the best abstract art.

The illustration shows one of the "cyclides of Dupin", a class of surfaces whose lines of curvature are all circles or straight lines. Photographs of 132 such models, with mathematical commentary, have been collected into a two-volume work, Mathematical Models, edited by Gerd Fischer and published by Vieweg, Wiesbaden, FRG, as part of their two-hundredth anniversary celebrations. In Britain. the books are distributed by Wiley; in the United States, by International Publishing Services, PO Box 230, Accord, Massachusetts 02018. 